\title{
Research \\ Primary amenorrhoea in Kashmiri adolescent females - a hospital based study
}

\author{
Shubana Rasool ${ }^{1}$, Aijaz Hakeem ${ }^{1}$, H Shafi ${ }^{1}$, Shahida M Mufti ${ }^{1}$ \\ Sri Lanka Journal of Obstetrics and Gynaecology 2010; 32: 53-56
}

\begin{abstract}
Objectives: The basic aim of this study was to evaluate various causes of primary amenorrhea in Kashmiri adolescent females.

Methods and material: the study was conducted in a tertiary care hospital on adolescent females with primary amenorrhoea in the age group of 13-19 years. A detailed history was taken and a thorough examination was done to note the secondary sexual characteristics. All cases were subjected to following investigations haemoglobin, total leukocyte count, differential leukocyte count, erythrocyte sedimentation rate, coagulation profile and hormonal profile. Radiological investigations and karyotyping were done in selected cases.
\end{abstract}

Statistical analysis: Done by descriptive analysis.

Results: Eugonadism was the commonest type of primary amenorrhoea and was seen in 68 cases. Imperforate hymen was seen in 28 cases and complete vaginal atresia in 16 . Among hypogonadotropic hypogonadism (23 cases) constitutional delay was seen in 15 cases, 8 cases diagnosed as hypogonadotropic hypogonadism was in fact having thyroid dysfunction. 3 cases with hypergonadotropic hypogonadism were seen and all were diagnosed as Turners syndrome.

Conclusions: Though mullerian abnormalities are the most common cause of primary amenorrhoea, thyroid dysfunction should be taken as a possibility in evaluating primary amenorrhoea in an iodine deficient belt like Kashmir.

Key words: adolescent, primary amenorrhoea, mullarian anomalies.

\footnotetext{
${ }^{1}$ Department of Obstetrics and Gynaecology, SK Institute of Medical Sciences, Srinagar, Kashmir, India.

Correspondence: Shubana Rasool

E-mail: aijazhakeem@yahoo.com
}

\section{Introduction}

Gynaecological disorders during childhood and adolescence have gained increasing attention not only in the western world but also in developing countries. In the past, teenage girls would hardly consult a gynaecologist. Now, more and more mothers take their daughters to specialists for treatment of genital disorders ${ }^{1}$. Menstrual disorders are the commonest gynaecological problem in adolescent girls. These range from amenorrhoea (both primary and secondary) to menorrhagia ${ }^{2}$. Obstructive anomalies of the female reproductive tract preclude the outflow of menstruation and may allow the collection of blood in the uterus and/or vagina and increase the likelihood of retrograde flow. These conditions may result in pelvic masses, endometriosis and/or pain ${ }^{3}$. The primary diagnostic technique for evaluating pelvic masses in adolescents is ultrasonography (USG). For cases, in which suspected diagnosis is appendicitis or another non gynaecologic condition or if the results are inconclusive, computed tomography (CT) and magnetic resonance imaging (MRI) may be helpful ${ }^{4}$. Adolescents with gonadal dysgenesis or androgen insensitivity may have abnormal pubertal development and primary amenorrhoea. The American College of Obstetricians and Gynaecologists recommends that young women schedule their first visit to obstetrician/gynecologist between the ages of 13 and 15 years ${ }^{5}$.

\section{Materials and methods}

This study was conducted in Lalla Ded Hospital, Government Medical College, Srinagar, India on adolescent females who attended the out patient department with primary amenorrhoea in the age group of 13 to 19 years over a period of one year.

After explaining the purpose of the study informed consent was obtained. After a detailed history, a thorough examination was done in the presence of a guardian/parent/female attendant in a place where privacy and confidentiality of the patient was maintained. The examination commenced with patients' general physical examination with focus on vitals (pulse rate in beats/minute, blood pressure in 
$\mathrm{mmHg}$ and respiratory rate in cycles/minute), pallor, height in centimetres, and weight in kilograms. All patients were subjected to systemic examination with particular attention on cardiovascular system, respiratory system and abdominal examination.

Each patient's secondary sexual characteristics were noted. Breast development (grading as per the Tanners scale of sexual maturation), axillary hair, pubic hair (grading as per Tanners scale of sexual maturation), external genitalia and gynaecological examination including vulval inspection, per rectal examination and per vaginal examination were done wherever necessary after the consent of the guardian/ parent.

All the adolescent females with primary ammenorrhoea were subjected to the routine investigations such as haemoglobin, total leucocyte count, differential leukocyte count, erythrocyte sedimentation rate and coagulation profile. Follicle stimulating hormone, leutnising hormone, thyroid stimulating hormone and serum prolactin levels were done in all cases. Serum testosterone, estradiol, 17$\mathrm{OH}$-progesterone levels and karyotyping were done in selected cases. Transabdominal ultrasound was done in all cases, transperineal and transrectal sonography was done in cases with mullerian anomalies. Computed tomography (CT) was done on spiral CT scan (WiproGE) in cases of mullerian abnormality. Magnetic resonance imaging (MRI) on Siemens helium cooled symphony 1.5 tesla MRI was done in patients with hyperprolactinaemia and mullerian anomalies.

Statistical analysis was done by descriptive statistical procedure.

\section{Results}

The commonest age of presentation in these cases was $15-16$ years $(48.9 \%)$, and the presenting complaint was primary amenorrhoea in $68(72.3 \%)$, primary amenorrhoea with abdominal pain in $14(14.8 \% \%)$, and primary amenorrhoea with abdominal lump in $12(12.8 \%)$.

In patients with eugonadism (68) imperforate hymen was diagnosed in 28 (41\%) (Figure 1), complete vaginal atresia with well formed uterus and fallopian tubes were found in 16 (24\%), vaginal septum in 5 (7\%) (Figure 2), 13 (19\%) complete absence of uterus and cervix but well formed vagina, 4 (6\%) with cervical and vaginal atresia (Figure 4 ) with well formed uterine body, 2 (3\%) uterine didelphys (Figure 3) with upper vaginal atresia which was subsequently confirmed on magnetic resonance imaging (MRI).

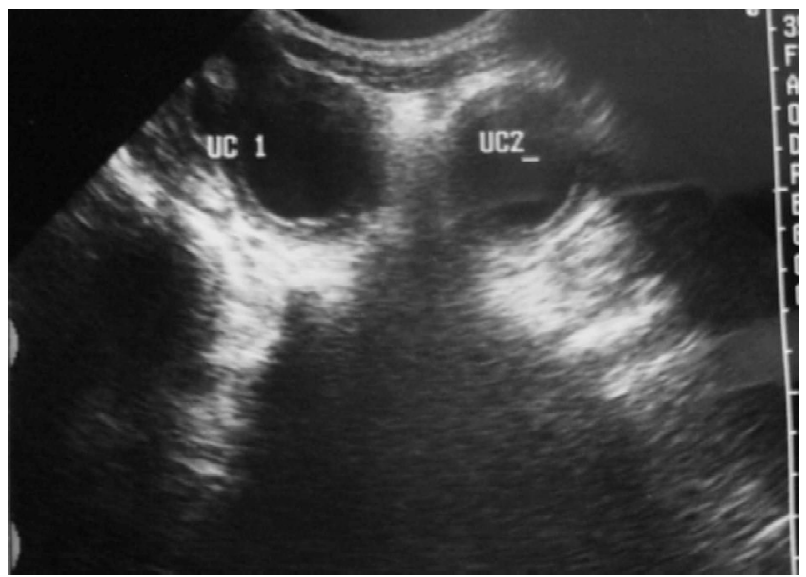

Figure 3 


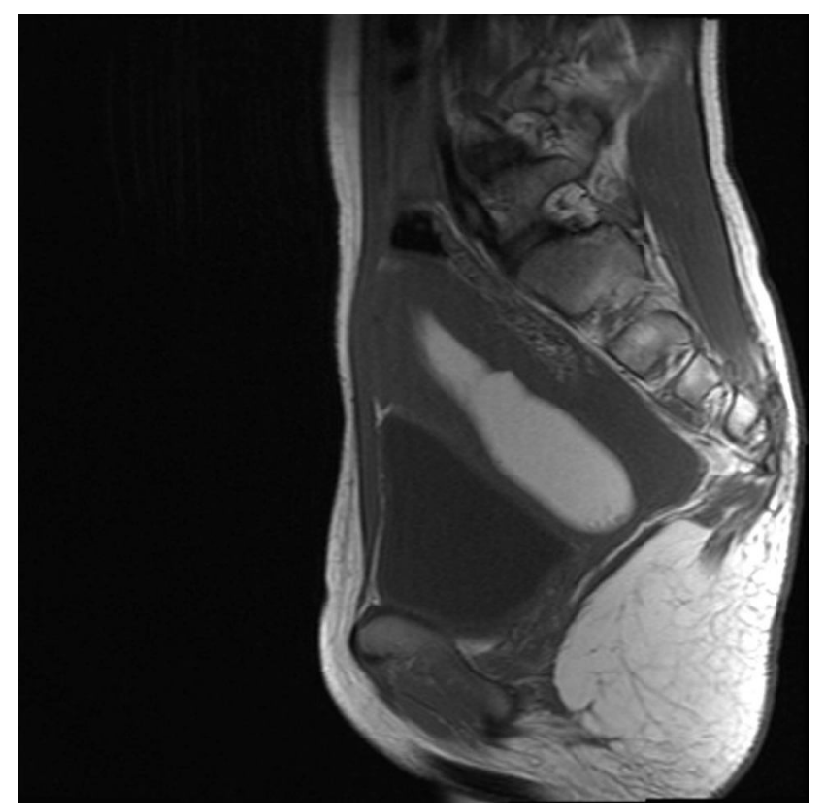

Figure 4

Among hypogonadotropic hypogonadism (23) constitutional delay was the leading cause 15 (65\%). This was diagnosed only after careful evaluation to exclude other causes of delayed puberty. Significant family history was present among $8(53 \%)$ cases. 8 (35\%) patients in this group were diagnosed to be having thyroid dysfunction.

3 cases with hypergonadotropic hypogonadism were seen during this study and all were diagnosed as Turners syndrome which was confirmed by karyotyping. On systemic examination 2 (67\%) cases with Turner's syndrome had cardiovascular system (CVS) abnormality.

\section{Discussion}

There are several available classifications of amenorrhoea and Reindollar's classification ${ }^{6}$ was used for our study. According to this classification primary amenorrhoea is classified as hypogonadotropic hypogonadism, hypergonadotropic hypogondism and eugonadism.

In our study eugonadism was the commonest type of primary amenorrhoea comprising of 68 (72.3\%) cases. MR imaging is useful in the work up of patients who present with primary amenorrhoea both for accurate diagnosis of pathologic conditions and for surgical planning ${ }^{7}$.

Rattanachaiyanont $\mathrm{M}$ et al (1997) ${ }^{8}$ in their study on 110 adolescents with primary amenorrhoea showed mullerian agenesis in $40 \%$ of cases. Mondal SK et al
$(2002)^{9}$ showed that mullerian duct abnormalities were present in $38 \%$ cases of primary amenorrhoea, gonadal agenesis in $18 \%$, Turner stigmata in $25 \%$. Rao K and Pillai NV $(1991)^{10}$ in their study showed that $50 \%$ cases of primary amenorrhoea were of muellerian dysgenesis.

In hypergonadotropic amenorrhoea there is elevated levels of LH and FSH due to gonadal failure. The causes include abnormal $X$ chromosome, sex chromosome mosaicism, environment and therapeutic ovarian toxins, enzyme deficiencies like congenital lipoid adrenal hyperplasia, $17 \alpha$-hydroxylase and 17, 20-desmolase deficiency, and aromatase deficiency. Turner's syndrome is the most common chromosomal abnormality causing gonadal failure. The associated stigmata of the Turners syndrome include short stature, webbed neck, shield chest and cubitus vulgus. In this study Turners syndrome was the only abnormality in hypergonadotropic amenorrhoea. 3 (3\%) cases with hypergonadotropic hypogonadism were seen and all were diagnosed as Turners syndrome which was confirmed by karyotyping. On systemic examination $2(68 \%)$ cases with Turner's syndrome had cardiovascular system (CVS) abnormality. Kumar A and Mittal S (1998) $)^{11}$ showed that out of 48 cases of primary amenorrhoea, $54 \%$ had mullerian anomalies, $23 \%$ had hypogonadotropic hypogonadism, 17\% had hypergonadotropic hypogonadism. Our study comprised lesser number of cases with hypergonadotropic hypogonsadism and the reason being as most such cases report to endocrinologists rather than to gynaecologists.

In hypogonadotropic amenorrhoea the pulsatile secretion of gonadotropins is suppressed below normal resulting in decreases in follicular development. These adolescents are in chronically low estrogenic levels. This type of amenorrhoea is associated with, constitutional delay, stress, eating disorders, excessive athletic training etc.

Among hypogonadotropic hypogonadism (23 cases) constitutional delay was the leading cause 15 $(65 \%)$ cases, they were diagnosed only after careful evaluation to exclude other causes of delayed puberty and with normal sexual development. 8 (35\%) cases in hypogonadotropic hypogonadism were diagnosed to be having thyroid dysfunction. Grubb MR et $\mathrm{al}^{12}$ had shown that between $25 \%$ and $40 \%$ of women with hypothyroidism are oligomenorrheic or amenorrheic. The adolescents in this region lie in an endemic belt $t^{13}$ and need to be evaluated for thyroid disorders if present with primary amenorrhoea. 


\section{Conclusions}

This prospective study was designed to look for various causes of primary amenorrhoea in adolescent females in Kashmir valley. The study comprised adolescents of 13 to 19 years of age. The most common age group that presented to us was $15-16$ years. Mean age of menarche in our cases was 12.8 years. Mullerian abnormality was the most common cause of primary amenorrhoea. However, thyroid dysfunction as a possible cause of primary amenorrhoea should be kept in mind when evaluating patients in an iodine deficient belt like Kashmir.

\section{References}

1. Chowdhury NNR, Sanyal M. Gynaecological problems in childhood and adolescence. J Indian M A 1975; 65(6): 179-83.

2. Sebanti G, Dutta R, Sibani S. A profile of adolescent girls with gynaecological problems. J Obstet Gynecol India 2005; 55(4): 353-5.

3. Breech LL, Laufer MR. Obstructive anomalies of the female reproductive tract. J Reprod Med 1999; 44(3): 233-40.

4. Schwartz LB, Seifer DB. Diagnostic imaging of adnexal masses: a review. J Reprod Med 1992; 37: 63 -71.

5. So-Young Shin, You-Young Lee, Sook-Young Yang, ByungKoo Yoon DukSoo Bae, DooSeok Choi. Characteristics of menstruation-related problems for adolescents and premarital women in Korea. European Journal of Obstetrics and Gynaecology and Reproductive Biology 2005; 121: 236-42.

6. Reindollar RH, Novak M, Tho SP, McDonough PG. Adult onset amenorrhoea - a study of 262 patients. Am J Obstet Gynecol 1986; 155: 531-43.

7. Caroline R, Hedvig H, Rosemarie F, Susan MA, Patrice MB, Meyer WR, Richard CS. Primary amenorrhoea: evaluation with MR Imaging. Radiology 1997; 203: 383-90.

8. Rattanachaiyanont M, Kunathikom S, Angsuwattana S, Techatraisak K, Mekmahan O, Karavagul C, Ko-AnantakulS. Primary ammenorhoea: a retrospective study at Siriraj Hospital. J Med Assoc Thai 1997; 80(10): 619-25.

9. Mondal SK, Guha D, Banerjee D, Sinha SK. Study of primary ammenorhoea with special reference to cytogenetic evaluation. Indian J Pathol Microbiol 2002; 45(2): 155-9.

10. Rao K, Pillai NV. Primary amenorrhoea (analysis of 40 cases). J Indian Med Assoc 1991; 89(2): 42-3.

11. Kumar A, Mittal S. Primary ammenorrhoea: analysis of 48 cases. J Indian Med Assoc 1998; 96(4): 119-20.

12. Grubb MR, Chakeres D, Malarkey WB. Patients with primary hypothyroidism presenting as prolactinomas. Am J Med 1987; 83: 765-9.

13. WHO. Report of a seminar on goitre control, New Delhi, WHO, SEARO, 1967. 